\title{
Microlocal Lefschetz classes of graph trace kernels
}

\author{
Yuichi IKE
}

April 18, 2018

\begin{abstract}
In this paper, we define the notion of graph trace kernels as a generalization of trace kernels. We associate a microlocal Lefschetz class with a graph trace kernel and prove that this class is functorial with respect to the composition of kernels. We apply graph trace kernels to the microlocal Lefschetz fixed point formula for constructible sheaves.
\end{abstract}

\section{Contents}

1 Introduction 1

2 Preliminaries 3

2.1 Review on sheaves ..................... 3

2.2 Compositions of kernels ...................... 4

3 Definition of graph trace kernels

3.1 Microlocal homology associated with morphisms . . . . . . . . . 7

3.2 Microlocal Lefschetz classes of graph trace kernels . . . . . . . . . . . 9

4 Main results 11

4.1 Compositions of microlocal Lefschetz classes . . . . . . . . . . . . . . 11

4.2 Operations on microlocal Lefschetz classes . . . . . . . . . . . . . . 14

4.3 Application to Lefschetz fixed point formula for constructible sheaves 17

$\begin{array}{ll}\text { References } & 18\end{array}$

\section{Introduction}

In [KS14], Kashiwara and Schapira introduced the notion of trace kernels and the method to associate a microlocal Euler class with a trace kernel.

Let $X$ be a $C^{\infty}$-manifold and $k$ be a field. We denote by $\omega_{X}$ the dualizing complex on $X$, that is, $\omega_{X} \simeq \operatorname{or}_{X}\left[d_{X}\right]$ where or $_{X}$ is the orientation sheaf on $X$ and $d_{X}$ is the dimension of $X$. Denote by $k_{\Delta_{X}}$ and $\omega_{\Delta_{X}}$ the direct image of $k_{X}$ and $\omega_{X}$ respectively under the diagonal embedding $\delta: X \hookrightarrow X \times X$. Let $\pi: T^{*} X \rightarrow X$ be the cotangent bundle of $X$. 
A trace kernel on $X$ is a triplet $(K, u, v)$ where $K$ is an object of the derived category of sheaves $\mathrm{D}^{\mathrm{b}}\left(k_{X \times X}\right)$ and $u, v$ are morphisms

$$
u: k_{\Delta_{X}} \longrightarrow K, v: K \longrightarrow \omega_{\Delta_{X}} .
$$

One can naturally define the microlocal Euler class $\mu \mathrm{eu}(K, u, v)$ as an element of $H_{\Lambda}^{0}\left(T^{*} X ; \mu h o m\left(k_{\Delta_{X}}, \omega_{\Delta_{X}}\right)\right) \simeq H_{\Lambda}^{0}\left(T^{*} X ; \pi^{-1} \omega_{X}\right)$, where $\Lambda=\operatorname{SS}(K) \cap T_{\Delta_{X}}^{*}(X \times X)$. Kashiwara and Schapira proved the functoriality of the microlocal Euler classes: the microlocal Euler class of the composition $K_{1} \circ K_{2}$ of two trace kernels is the composition of the microlocal Euler classes of $K_{1}$ and $K_{2}$ [KS14, Theorem 6.3].

On the other hand, microlocal Lefschetz classes of elliptic pairs (Guillermou Gu96]) and Lefschetz cycles of constructible sheaves (Matsui-Takeuchi [MT10]) were introduced in order to prove the microlocal fixed point formula. For elliptic pairs, see Schapira-Schneiders [ScSn94]. For recent results on this subject, see also [IMT15] and [RTT13].

Let us recall the notion of Lefschetz cycles defined in [MT10]. Let $X$ be a real analytic manifold and $\phi: X \rightarrow X$ be a morphism of manifolds. We denote by $\mathrm{D}_{\mathbb{R} \text {-c }}^{\mathrm{b}}\left(k_{X}\right)$ the bounded derived category of $\mathbb{R}$-constructible sheaves on $X$. Denote by $\omega_{\Gamma_{\phi}}$ the direct image of $\omega_{X}$ under the graph map $\delta_{\phi}: X \hookrightarrow X \times X, x \mapsto(x, \phi(x))$. With a pair $(F, \Phi)$ of $F \in \mathrm{D}_{\mathbb{R}-\mathrm{c}}^{\mathrm{b}}\left(k_{X}\right)$ and $\Phi \in \operatorname{Hom}\left(\phi^{-1} F, F\right)$, one can associate a cohomology class $\mu \operatorname{Le}(F, \Phi, \phi) \in H_{\Lambda}^{0}\left(T^{*} X ; \mu_{\Delta_{X}}\left(\omega_{\Gamma_{\phi}}\right)\right)$, where $\Lambda:=\operatorname{SS}(F) \cap T_{\Delta_{X}}^{*}(X \times$ $X) \cap T_{\Gamma_{\phi}}^{*}(X \times X)$. This class is called the Lefschetz cycle or the microlocal Lefschetz class of the pair $(F, \Phi)$.

The microlocal Lefschetz classes of $\mathbb{R}$-constructible sheaves can be treated in the same way as the microlocal Euler classes of trace kernels. Define $\mathrm{D}_{X} F:=$ $\mathrm{R} \mathscr{H} o m\left(F, \omega_{X}\right)$, the dual of $F$. The pair $(F, \Phi)$ gives natural morphisms

$$
k_{\Delta_{X}} \longrightarrow \mathrm{D}_{X} F \otimes F \longrightarrow \omega_{\Gamma_{\phi}} .
$$

The composition of the above morphisms defines a cohomology class in $H_{\Lambda}^{0}\left(T^{*} X ; \mu h o m\left(k_{\Delta_{X}}, \omega_{\Gamma_{\phi}}\right)\right) \simeq H_{\Lambda}^{0}\left(T^{*} X ; \mu_{\Delta_{X}}\left(\omega_{\Gamma_{\phi}}\right)\right)$ and this class coincides with $\mu \operatorname{Le}(F, \Phi, \phi)$.

In this paper, we extend the notion of trace kernels so that we can treat fixed point formulas. Then we associate a microlocal Lefschetz class with such a kernel and prove the functoriality of the class.

For a $C^{\infty}$-manifold $X$ and a morphism of manifolds $\phi: X \rightarrow X$, a $\phi$-graph trace kernel is a triplet $(K, u, v)$ where $K \in \mathrm{D}^{\mathrm{b}}\left(k_{X \times X}\right)$ and $u, v$ are morphisms

$$
u: k_{\Delta_{X}} \longrightarrow K, v: K \longrightarrow \omega_{\Gamma_{\phi}} .
$$

One defines the microlocal Lefschetz class $\mu \operatorname{Le}(K, u, v, \phi)$ as an element of $H_{\Lambda}^{0}\left(T^{*} X ; \mu h o m\left(k_{\Delta_{X}}, \omega_{\Gamma_{\phi}}\right)\right)$, where $\Lambda:=\operatorname{SS}(K) \cap T_{\Delta_{X}}^{*}(X \times X) \cap T_{\Gamma_{\phi}}^{*}(X \times X)$. By (1.2), a pair $(F, \Phi)$ of $F \in \mathrm{D}_{\mathbb{R}-\mathrm{c}}^{\mathrm{b}}(X)$ and $\Phi \in \operatorname{Hom}\left(\phi^{-1} F, F\right)$ defines a $\phi$-graph trace kernel.

Our main result is the functoriality of microlocal Lefschetz classes: the microlocal Lefschetz class of the composition $K_{1} \circ K_{2}$ of two graph trace kernels is the 
composition of microlocal Lefschetz classes of $K_{1}$ and $K_{2}$ (for a more precise statement, see Theorem 4.3). As an application, we prove the microlocal Lefschetz fixed point formula for constructible sheaves.

Finally, let us explain the difference between our construction and that of [KS14]. In the last section of [KS14, the authors have remarked that trace kernels can be adapted to the Lefschetz fixed point formula for constructible sheaves. However, their construction is not suitable to prove the functoriality of the cohomology classes. Therefore, we extend the notion of trace kernels itself and prove the functoriality by using the new framework.

\section{Preliminaries}

\subsection{Review on sheaves}

In this paper, all manifolds are assumed to be real manifolds of class $C^{\infty}$. Throughout this paper, let $k$ be a field of characteristic zero. We follow the notation of [KS90].

Let $X$ be a manifold. We denote by $\pi_{X}: T^{*} X \rightarrow X$ its cotangent bundle. If there is no risk of confusion, we simply write $\pi$ instead of $\pi_{X}$. For a submanifold $M$ of $X$, we denote by $T_{M}^{*} X$ the conormal bundle to $M$. In particular, $T_{X}^{*} X$ denotes the zero-section of $T^{*} X$. We also denote by $a: T^{*} X \rightarrow T^{*} X$ the antipodal map defined by $(x ; \xi) \mapsto(x ;-\xi)$. A set $\Lambda \subset T^{*} X$ is said to be conic if it is invariant by the action of $\mathbb{R}^{+}$on $T^{*} X$.

Let $f: X \rightarrow Y$ be a morphism of manifolds. With $f$ one associates the maps

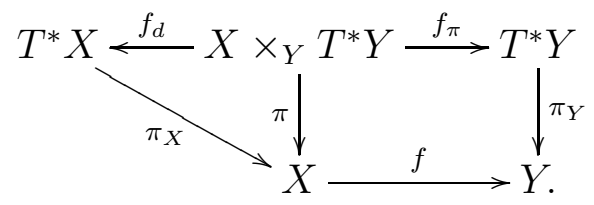

We denote by $k_{X}$ the constant sheaf on $X$ with stalk $k$ and by $\mathrm{D}^{\mathrm{b}}\left(k_{X}\right)$ the bounded derived category of sheaves of $k$-vector spaces on $X$. One can define Grothendieck's six operations $\mathrm{R} f_{*}, f^{-1}, \mathrm{R} f_{!}, f^{!}, \stackrel{\mathrm{L}}{\otimes}, \mathrm{R} \mathscr{H}$ om as functors of derived categories of sheaves. Since the functor $\cdot \underset{k_{X}}{\otimes}$. is exact, we simply write $\otimes$ instead of $\stackrel{\mathrm{L}}{\otimes}$. One denotes by $\omega_{X}$ the dualizing complex on $X$. That is, if $\mathbf{a}_{X}: X \rightarrow$ pt denotes the natural map, set $\omega_{X}:=\mathbf{a}_{X}^{!} k_{\mathrm{pt}}$. One also denotes by $\omega_{X}^{\otimes-1}:=\mathrm{R} \mathscr{H}$ om $\left(\omega_{X}, k_{X}\right)$ the dual of $\omega_{X}$. More generally, for a morphism $f: X \rightarrow Y$, we denote by $\omega_{X / Y}:=$ $f^{!} k_{Y} \simeq \omega_{X} \otimes f^{-1} \omega_{Y}^{\otimes-1}$ the relative dualizing complex. Note that $\omega_{X} \simeq \operatorname{or}_{X}\left[d_{X}\right]$, where or $_{X}$ is the orientation sheaf on $X$ and $d_{X}$ is the dimension of $X$. Recall that there is a natural morphism of functors

$$
\omega_{X / Y} \otimes f^{-1}(\cdot) \rightarrow f^{!}(\cdot) .
$$

We define the duality functor by

$$
\mathrm{D}_{X} F:=\mathrm{R} \mathscr{H} o m\left(F, \omega_{X}\right) .
$$


For $F \in \mathrm{D}^{\mathrm{b}}\left(k_{X}\right)$, we denote by $\operatorname{Supp}(F)$ the support of $F$ and by $\operatorname{SS}(F)$ its micro-support, a closed conic involutive subset of $T^{*} X$.

For a closed submanifold $M$ of $X$, one denotes by $\mu_{M}: \mathrm{D}^{\mathrm{b}}\left(k_{X}\right) \rightarrow \mathrm{D}_{\mathbb{R}^{+}}^{\mathrm{b}}\left(k_{T_{M}^{*} X}\right)$ Sato's microlocalization functor along $M$, where $\mathrm{D}_{\mathbb{R}^{+}}^{\mathrm{b}}\left(k_{T_{M}^{*} X}\right)$ is the full subcategory of $\mathrm{D}^{\mathrm{b}}\left(k_{T_{M}^{*} X}\right)$ consisting of $\mathbb{R}^{+}$-conic objects. We shall use the functor $\mu$ hom defined in [KS90]. For $F_{1}, F_{2} \in \mathrm{D}^{\mathrm{b}}\left(k_{X}\right)$, one defines the bifunctor

$$
\begin{aligned}
& \text { uhom: } \mathrm{D}^{\mathrm{b}}\left(k_{X}\right)^{\mathrm{op}} \times \mathrm{D}^{\mathrm{b}}\left(k_{X}\right) \rightarrow \mathrm{D}_{\mathbb{R}^{+}}^{\mathrm{b}}\left(k_{T^{*} X}\right) \\
& \mu h o m\left(F_{1}, F_{2}\right):=\mu_{\Delta} \mathrm{R} \mathscr{H o m}\left(q_{2}^{-1} F_{1}, q_{1}^{!} F_{2}\right) \text {, }
\end{aligned}
$$

where $q_{1}$ and $q_{2}$ are the first and second projections from $X \times X$ and $\Delta$ is the diagonal. Note that the support of $\mu h o m\left(F_{1}, F_{2}\right)$ satisfies

$$
\operatorname{Supp}\left(\mu h o m\left(F_{1}, F_{2}\right)\right) \subset \mathrm{SS}\left(F_{1}\right) \cap \mathrm{SS}\left(F_{2}\right) \text {. }
$$

Furthermore, we have the isomorphism

$$
\left.\operatorname{R} \pi_{*} \mu h o m\left(F_{1}, F_{2}\right) \simeq \mu h o m\left(F_{1}, F_{2}\right)\right|_{X} \simeq \operatorname{R} \mathscr{H o m}\left(F_{1}, F_{2}\right) .
$$

\subsection{Compositions of kernels}

We follow the notation of [KS14]. The results in this subsection are the same as in Section 3 of [KS14]. For the convenience of the readers, we give proofs of these results here.

Notation 2.1. (i) For a manifold $X$, we denote by $\delta: X \hookrightarrow X \times X$ the diagonal embedding and by $\Delta_{X}$ the diagonal set of $X \times X$.

(ii) Let $X_{i}(i=1,2,3)$ be manifolds. For short, we write $X_{i j}:=X_{i} \times X_{j}, X_{123}:=$ $X_{1} \times X_{2} \times X_{3}, X_{11223}:=X_{1} \times X_{1} \times X_{2} \times X_{2} \times X_{3}$, etc.

(iii) Let $\phi_{i}: X_{i} \rightarrow X_{i}(i=1,2,3)$ be morphisms of manifolds. We write $\phi_{i j}:=$ $\phi_{i} \times \phi_{j}: X_{i j} \rightarrow X_{i j}$.

(iv) For simplicity, we shall write $k_{i}$ instead of $k_{X_{i}}$ and $\omega_{i}$ instead of $\omega_{X_{i}}$, etc. We also write $k_{\Delta_{i}}$ instead of $k_{\Delta_{X_{i}}}$.

(v) We denote by $\pi_{i}$ or $\pi_{i j}$, etc. the projection $T^{*} X_{i} \rightarrow X_{i}$ or $T^{*} X_{i j} \rightarrow X_{i j}$, etc.

(vi) We use the same symbol $q_{i}$ for the projections $X_{i j} \rightarrow X_{i}$ and $X_{123} \rightarrow X_{i}$. We also denote by $q_{i j}$ the projection $X_{123} \rightarrow X_{i j}$, by $p_{i}$ the projection $T^{*} X_{i j} \rightarrow$ $T^{*} X_{i}$, and by $p_{i j}$ the projection $T^{*} X_{123} \rightarrow T^{*} X_{i j}$.

(vii) We denote by $p_{j^{a}}$ (resp. $p_{i j^{a}}$ ) the composition of $p_{j}$ (resp. $p_{i j}$ ) and the antipodal map on $T^{*} X_{j}$.

(viii) We denote by $\delta_{2}$ the diagonal embedding $X_{123} \rightarrow X_{1223}$.

Recall the operations of composition of kernels defined in [KS14]. 
Definition 2.2 ([KS14] $)$. We define the operations of composition of kernels as follows:

$$
\begin{aligned}
\stackrel{\circ}{\circ}: \mathrm{D}^{\mathrm{b}}\left(k_{12}\right) \times \mathrm{D}^{\mathrm{b}}\left(k_{23}\right) & \rightarrow \mathrm{D}^{\mathrm{b}}\left(k_{13}\right) \\
\left(K_{12}, K_{23}\right) \mapsto K_{12} \stackrel{\circ}{\circ} K_{23}: & : \mathrm{R} q_{13 !}\left(q_{12}^{-1} K_{12} \otimes q_{23}^{-1} K_{23}\right) \\
& \simeq \mathrm{R} q_{13 !} \delta_{2}^{-1}\left(K_{12} \otimes K_{23}\right), \\
\underset{2}{*}: \mathrm{D}^{\mathrm{b}}\left(k_{12}\right) \times \mathrm{D}^{\mathrm{b}}\left(k_{23}\right) \rightarrow \mathrm{D}^{\mathrm{b}}\left(k_{13}\right) & \\
\left(K_{12}, K_{23}\right) & \mapsto K_{12}^{*} K_{23}:=\mathrm{R} q_{13 *}\left(\omega_{X_{123} / X_{1223}}^{\otimes-1} \otimes \delta_{2}^{!}\left(K_{12} \otimes K_{23}\right)\right) .
\end{aligned}
$$

By (2.1), we have a natural morphism $\delta_{2}^{-1}(\cdot) \rightarrow \omega_{X_{123} / X_{1223}}^{\otimes-1} \otimes \delta_{2}^{!}(\cdot)$. Combining this with the morphism $\mathrm{R} q_{13 !} \rightarrow \mathrm{R} q_{13 *}$, we obtain a natural morphism

$$
K_{12} \underset{2}{\circ} K_{23} \rightarrow K_{12} \underset{2}{*} K_{23}
$$

This is an isomorphism if $p_{12^{a}}^{-1}\left(\operatorname{SS}\left(K_{12}\right)\right) \cap p_{23}^{-1}\left(\mathrm{SS}\left(K_{23}\right)\right)$ is proper over $T^{*} X_{13}$.

We define the composition of kernels on cotangent bundles.

Definition 2.3 ([KS14] $)$. For kernels on cotangent bundles, we define the composition of kernels as follows:

$$
\begin{aligned}
\stackrel{a}{\circ}: \mathrm{D}^{\mathrm{b}}\left(k_{T^{*} X_{12}}\right) \times \mathrm{D}^{\mathrm{b}}\left(k_{T^{*} X_{23}}\right) & \rightarrow \mathrm{D}^{\mathrm{b}}\left(k_{T^{*} X_{13}}\right) \\
\left(K_{12}, K_{23}\right) & \mapsto K_{12} \underset{2}{\stackrel{a}{\circ}} K_{23}:=\mathrm{R} p_{13 !}\left(p_{12^{a}}^{-1} K_{12} \otimes p_{23}^{-1} K_{23}\right) .
\end{aligned}
$$

We also define the corresponding operations for subsets of cotangent bundles. Let $A \subset T^{*} X_{12}$ and $B \subset T^{*} X_{23}$. We set

$$
\begin{aligned}
& A \stackrel{a}{\underset{2}{\times}} B:=p_{12^{a}}^{-1}(A) \cap p_{23}^{-1}(B), \\
& \stackrel{2}{\stackrel{a}{\circ}} B_{2}:=p_{13}(A \underset{2}{\stackrel{a}{\times}} B) .
\end{aligned}
$$

In order to define a composition morphism, we need the following lemma. Let $X, Y, S$ be manifolds. Let $q_{X}: X \rightarrow S$ and $q_{Y}: Y \rightarrow S$ be morphisms. Assume that

$$
X \times{ }_{S} Y \text { is a submanifold of } X \times Y \text {. }
$$

Let $j$ be an embedding $X \times{ }_{S} Y \hookrightarrow X \times Y$. Noticing that $\left(X \times{ }_{S} Y\right) \times(X \times Y) T^{*}(X \times Y) \simeq$ $T^{*} X \times{ }_{S} T^{*} Y$, we have the following morphisms:

$$
T^{*}\left(X \times_{S} Y\right) \stackrel{j_{d}}{\longleftarrow} T^{*} X \times_{S} T^{*} Y \stackrel{j_{\pi}}{\longrightarrow} T^{*} X \times T^{*} Y .
$$

Lemma 2.4. (cf. [KS90, Proposition 4.4.8]) For $F_{1}, G_{1} \in \mathrm{D}^{\mathrm{b}}\left(k_{X}\right)$ and $F_{2}, G_{2} \in$ $\mathrm{D}^{\mathrm{b}}\left(k_{Y}\right)$, there is a canonical morphism

$$
\operatorname{R} j_{d !}\left(\mu h o m\left(G_{1}, F_{1}\right) \underset{S}{\otimes} \mu h o m\left(G_{2}, F_{2}\right)\right) \rightarrow \mu h o m\left(j^{!}\left(G_{1} \otimes G_{2}\right) \otimes \omega_{X \times{ }_{S} Y / X \times Y}^{\otimes-1}, F_{1} \underset{S}{\otimes} F_{2}\right) .
$$


Proof. First, we construct the morphism when $S=$ pt. By using the morphism $\mu_{M}(F) \otimes \mu_{N}(G) \rightarrow \mu_{M \times N}(F \otimes G)$ [KS90, Proposition 4.3.6], we obtain a chain of morphisms

$$
\begin{aligned}
& \mu_{\Delta_{X}} \mathrm{R} \mathscr{H} O m\left(q_{X_{2}}^{-1} G_{1}, q_{X} \stackrel{!}{1} F_{1}\right) \otimes \mu_{\Delta_{Y}} \mathrm{R} \mathscr{H} o m\left(q_{Y}{ }^{-1} G_{2}, q_{Y} ! F_{2}\right) \\
& \rightarrow \mu_{\Delta_{X} \times \Delta_{Y}}\left(\mathrm{R} \mathscr{H} o m\left(q_{X}{ }^{-1} G_{1}, q_{X}{ }_{1} F_{1}\right) \otimes \mathrm{R} \mathscr{H} o m\left(q_{Y}{ }^{-1} G_{2}, q_{Y}{ }_{1} F_{2}\right)\right) \\
& \rightarrow \mu_{\Delta_{X} \times \Delta_{Y}}\left(\mathrm{R} \mathscr{H} o m\left(q_{X}{ }^{-1} G_{1} \otimes q_{Y}{ }^{-1} G_{2}, q_{X}{ }_{1}^{!} F_{1} \otimes q_{Y} ! F_{2}\right)\right. \\
& \simeq \mu_{\Delta_{X \times Y}} \operatorname{R} \mathscr{H} o m\left(q_{2}^{-1}\left(G_{1} \otimes G_{2}\right), q_{1}^{!}\left(F_{1} \otimes F_{2}\right)\right) \text {. }
\end{aligned}
$$

Next, we treat the general case. Using the morphism

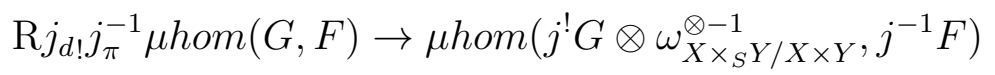

from [KS90, Proposition 4.4.7], we obtain a chain of morphisms

$$
\begin{aligned}
& \operatorname{R} j_{d !} j_{\pi}^{-1}\left(\mu h o m\left(G_{1}, F_{1}\right) \otimes \mu h o m\left(G_{2}, F_{2}\right)\right) \\
\rightarrow & \operatorname{R} j_{d !} j_{\pi}^{-1} \mu h o m\left(G_{1} \otimes G_{2}, F_{1} \otimes F_{2}\right) \\
\rightarrow & \mu h o m\left(j^{!}\left(G_{1} \otimes G_{2}\right) \otimes \omega_{X \times_{S} Y / X \times Y}^{\otimes-1}, j^{-1}\left(F_{1} \otimes F_{2}\right)\right) .
\end{aligned}
$$

Proposition 2.5 ([KS14]). For $G_{1}, F_{1} \in \mathrm{D}^{\mathrm{b}}\left(k_{12}\right)$ and $G_{2}, F_{2} \in \mathrm{D}^{\mathrm{b}}\left(k_{23}\right)$, there is a composition morphism:

$$
\mu h o m\left(G_{1}, F_{1}\right) \underset{2}{\stackrel{a}{\circ}} \mu h o m\left(G_{2}, F_{2}\right) \rightarrow \mu h o m\left(G_{1} \underset{2}{*} G_{2}, F_{1} \underset{2}{\circ} F_{2}\right) .
$$

Proof. We shall apply Lemma 2.4 for $X_{12} \rightarrow X_{2}$ and $X_{23} \rightarrow X_{2}$. In this case, $X_{12} \times_{X_{2}} X_{23} \simeq X_{123}$ and $j$ is the diagonal embedding $X_{123} \hookrightarrow X_{1223}$. Consider the following commutative diagram:

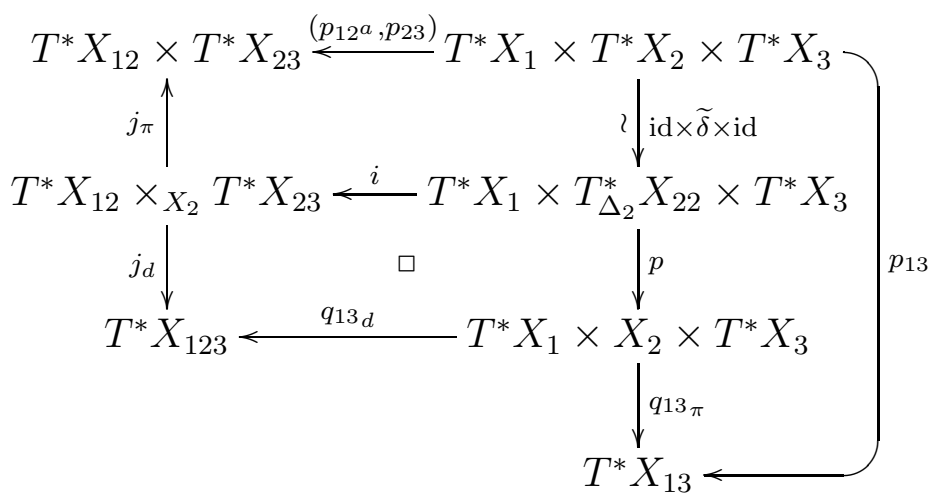

where $\widetilde{\delta}$ is defined by $\left(x_{2} ; \xi_{2}\right) \mapsto\left(x_{2}, x_{2} ;-\xi_{2}, \xi_{2}\right)$.

By Lemma 2.4, we get a morphism

$$
\begin{aligned}
& \mathrm{R} j_{d !} j_{\pi}^{-1}\left(\mu h o m\left(G_{1}, F_{1}\right) \otimes \mu h o m\left(G_{2}, F_{2}\right)\right) \\
& \rightarrow \mu h o m\left(j^{!}\left(G_{1} \otimes G_{2}\right) \otimes \omega_{X_{123} / X_{1223}}^{\otimes-1}, j^{-1}\left(F_{1} \otimes F_{2}\right)\right) .
\end{aligned}
$$


Set $G:=j^{!}\left(G_{1} \otimes G_{2}\right) \otimes \omega_{X_{123} / X_{1223}}^{\otimes-1} \in \mathrm{D}^{\mathrm{b}}\left(k_{123}\right)$ and $F:=j^{-1}\left(F_{1} \otimes F_{2}\right) \in \mathrm{D}^{\mathrm{b}}\left(k_{123}\right)$. Combining (2.11) with the morphism

$$
\mathrm{R} q_{13 \pi} q_{13}{ }_{d}^{-1} \mu h o m(G, F) \rightarrow \mu h o m\left(\mathrm{R} q_{13 *} G, \operatorname{R} q_{13 !} F\right)
$$

from [KS90, Proposition 4.4.7], we get a morphism

$$
\begin{aligned}
& \mathrm{R} q_{13 \pi !} q_{13}{ }_{d}^{-1} \mathrm{R} j_{d !} j_{\pi}^{-1}\left(\mu h o m\left(G_{1}, F_{1}\right) \otimes \mu h o m\left(G_{2}, F_{2}\right)\right) \rightarrow \mu h o m\left(\mathrm{R} q_{13 *} G, \mathrm{R} q_{13 !} F\right) \\
& \simeq \operatorname{\mu hom}\left(G_{1} \underset{2}{*} G_{2}, F_{1} \circ F_{2}\right) \text {. }
\end{aligned}
$$

By the above commutative diagram, we have

$$
\begin{aligned}
\mathrm{R} q_{13 \pi !} q_{13}{ }_{d}^{-1} \mathrm{R} j_{d !} j_{\pi}^{-1} & \simeq \mathrm{R} q_{13 \pi !} \mathrm{R} p_{!} i^{-1} j_{\pi}^{-1} \\
& \simeq \mathrm{R} q_{13 \pi !} \mathrm{R} p_{!}(\mathrm{id} \times \widetilde{\delta} \times \mathrm{id}) !\left(p_{12^{a}}, p_{23}\right)^{-1} \\
& \simeq \mathrm{R} p_{13 !}\left(p_{12^{a}}, p_{23}\right)^{-1}
\end{aligned}
$$

Thus, the result follows from the isomorphisms

$$
\begin{aligned}
& \operatorname{R} q_{13 \pi !} q_{13}{ }_{d}^{-1} \mathrm{R} j_{d !} j_{\pi}^{-1}\left(\mu h o m\left(G_{1}, F_{1}\right) \otimes \mu h o m\left(G_{2}, F_{2}\right)\right) \\
\simeq & \operatorname{Rp} p_{13 !}\left(p_{12^{a}}, p_{23}\right)^{-1}\left(\mu h o m\left(G_{1}, F_{1}\right) \otimes \mu h o m\left(G_{2}, F_{2}\right)\right) \\
\simeq & \operatorname{R} p_{13 !}\left(p_{12^{a}}^{-1} \mu h o m\left(G_{1}, F_{1}\right) \otimes p_{23}^{-1} \mu h o m\left(G_{2}, F_{2}\right)\right) .
\end{aligned}
$$

\section{Definition of graph trace kernels}

\subsection{Microlocal homology associated with morphisms}

Let $X$ be a manifold and $\phi: X \rightarrow X$ be a morphism of manifolds. We shall identify $X$ with the diagonal $\Delta_{X}$ of $X \times X$ and write $\Delta$ instead of $\Delta_{X}$ if there is no risk of confusion. We shall also identify $T^{*} X$ with $T_{\Delta}^{*}(X \times X)$ by means of the map

$$
\delta_{T^{*} X}^{a}: T^{*} X \rightarrow T^{*}(X \times X),(x ; \xi) \mapsto(x, x ; \xi,-\xi) .
$$

We denote by $\delta_{\phi}=\left(\operatorname{id}_{X}, \phi\right): X \hookrightarrow X \times X$ the graph map of $\phi$ and by $\Gamma_{\phi}=\delta_{\phi}(X)$ the graph of $\phi$. Set $k_{\Gamma_{\phi}}:=\left(\delta_{\phi}\right)_{*} k_{X}, \omega_{\Gamma_{\phi}}:=\left(\delta_{\phi}\right)_{*} \omega_{X}$ and $\omega_{\Gamma_{\phi}}^{\otimes-1}:=\left(\delta_{\phi}\right)_{*} \omega_{X}^{\otimes-1}$. In the case $\phi=\mathrm{id}_{X}$, we shall write $\delta$ for $\delta_{\phi}$ and $k_{\Delta}$ for $k_{\Gamma_{\phi}}$, etc.

Definition 3.1. Let $\Lambda$ be a closed conic subset of $T^{*} X$. We set

(i) $\mathscr{M} \mathscr{H}_{\Lambda}(\phi):=\mathrm{R} \Gamma_{\Lambda}\left(\delta_{T^{*} X}^{a}\right)^{-1} \mu h o m\left(k_{\Delta}, \omega_{\Gamma_{\phi}}\right)$,

(ii) $\mathbb{M H}_{\Lambda}(\phi):=\mathrm{R} \Gamma\left(T^{*} X ; \mathscr{M}_{\mathscr{H}_{\Lambda}}(\phi)\right)$,

(iii) $\mathbb{M H}_{\Lambda}^{n}(\phi):=H^{n}\left(\mathbb{M H}_{\Lambda}(\phi)\right)$.

Let $\phi_{i}: X_{i} \rightarrow X_{i}(i=1,2,3)$ be morphisms of manifolds. We write $\Delta_{i}$ for $\Delta_{X_{i}} \subset X_{i i}$, etc. 
Lemma 3.2. We have natural morphisms:

(i) $\omega_{\Gamma_{\phi_{12} 22}} \underset{\left({ }^{2}\right.}{\circ}\left(k_{\Gamma_{\phi_{2}}} \otimes \omega_{\Gamma_{\phi_{3}}}\right) \rightarrow \omega_{\Gamma_{\phi_{13}}}$,

(ii) $k_{\Delta_{13}} \rightarrow k_{\Delta_{12}} \underset{22}{*}\left(\omega_{\Delta_{2}}^{\otimes-1} \otimes k_{\Delta_{3}}\right)$.

Proof. We denote by $\delta_{22}$ the diagonal embedding $X_{112233} \hookrightarrow X_{11222233}$.

(i) We have morphisms

$$
\begin{aligned}
\omega_{\Gamma_{\phi_{12}} 22} \circ\left(k_{\Gamma_{\phi_{2}}} \otimes \omega_{\Gamma_{\phi_{3}}}\right) & =\operatorname{R} q_{1133 !} \delta_{22}^{-1}\left(\omega_{\Gamma_{\phi_{1}}} \otimes \omega_{\Gamma_{\phi_{2}}} \otimes k_{\Gamma_{\phi_{2}}} \otimes \omega_{\Gamma_{\phi_{3}}}\right) \\
& \simeq \operatorname{R} q_{1133 !}\left(\omega_{\Gamma_{\phi_{1}}} \otimes \omega_{\Gamma_{\phi_{2}}} \otimes \omega_{\Gamma_{\phi_{3}}}\right) \\
& \rightarrow \omega_{\Gamma_{\phi_{13}}} .
\end{aligned}
$$

(ii) The proof is the same as that of [KS14, Lemma 4.3].

Proposition 3.3. We have a natural composition morphism

$$
\mu h o m\left(k_{\Delta_{12}}, \omega_{\Gamma_{\phi_{12}}}\right) \stackrel{a}{\circ} \mu h o m\left(k_{\Delta_{23}}, \omega_{\Gamma_{\phi_{23}}}\right) \rightarrow \mu h o m\left(k_{\Delta_{13}}, \omega_{\Gamma_{\phi_{13}}}\right) .
$$

Proof. We have the following isomorphisms:

$$
\begin{aligned}
\mu h o m\left(k_{\Delta_{23}}, \omega_{\Gamma_{\phi_{23}}}\right) & \simeq \mu h o m\left(\left(\omega_{2}^{\otimes-1} \otimes k_{233}\right) \otimes k_{\Delta_{23}},\left(\omega_{2}^{\otimes-1} \otimes k_{233}\right) \otimes \omega_{\Gamma_{\phi_{23}}}\right) \\
& \simeq \mu h o m\left(\omega_{\Delta_{2}}^{\otimes-1} \otimes k_{\Delta_{3}}, k_{\Gamma_{\phi_{2}}} \otimes \omega_{\Gamma_{\phi_{3}}}\right) .
\end{aligned}
$$

Applying Proposition 2.5 and Lemma 3.2, we get a chain of morphisms

$$
\begin{aligned}
& \mu h o m\left(k_{\Delta_{12}}, \omega_{\Gamma_{\phi_{12}}}\right) \stackrel{a}{\circ} \mu h o m\left(k_{\Delta_{23}}, \omega_{\Gamma_{\phi_{23}}}\right) \\
& \simeq \operatorname{\mu hom}\left(k_{\Delta_{12}}, \omega_{\Gamma_{\phi_{12}}}\right) \stackrel{a}{\stackrel{a}{\circ}} \mu \operatorname{hom}\left(\omega_{\Delta_{2}}^{\otimes-1} \otimes k_{\Delta_{3}}, k_{\Gamma_{\phi_{2}}} \otimes \omega_{\Gamma_{\phi_{3}}}\right) \\
& \rightarrow \mu h o m\left(k_{\Delta_{12}}{ }_{22}^{*}\left(\omega_{\Delta_{2}}^{\otimes-1} \otimes k_{\Delta_{3}}\right), \omega_{\Gamma_{\phi_{12}}} \circ\left(k_{\Gamma_{\phi_{2}}} \otimes \omega_{\Gamma_{\phi_{3}}}\right)\right) \\
& \rightarrow \mu h o m\left(k_{\Delta_{13}}, \omega_{\Gamma_{\phi_{13}}}\right) \text {. }
\end{aligned}
$$

Corollary 3.4. Let $\Lambda_{i j}$ be a closed conic subset of $T^{*} X_{i j}(i j=12,23)$ and assume that

$$
\Lambda_{12} \underset{2}{\stackrel{a}{\times}} \Lambda_{23} \rightarrow T^{*} X_{13} \text { is proper. }
$$

Set $\Lambda_{13}:=\Lambda_{12} \stackrel{a}{\stackrel{a}{\circ}} \Lambda_{23} \cap\left(\delta_{T^{*} X_{13}}^{a}\right)^{-1}\left(T_{\Gamma_{\phi_{13}}}^{*} X_{1313}\right)$. The composition of kernels induces a morphism

$$
\stackrel{a}{\circ} \underset{2}{\circ} \mathbb{M H}_{\Lambda_{12}}\left(\phi_{12}\right) \otimes \mathbb{M H}_{\Lambda_{23}}\left(\phi_{23}\right) \rightarrow \mathbb{M H}_{\Lambda_{13}}\left(\phi_{13}\right) .
$$

In particular, a cohomology class $\lambda \in \mathbb{M H}_{\Lambda_{12}}^{0}\left(\phi_{12}\right)$ defines a morphism

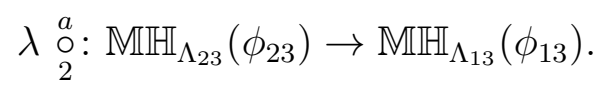


Proof. Noticing that

$$
\begin{aligned}
& \mathbb{M H}_{\Lambda_{12}}\left(\phi_{12}\right) \simeq \operatorname{R\Gamma }_{\delta_{T^{*} X_{12}}^{a} \Lambda_{12}}\left(T^{*} X_{1122} ; \mu h o m\left(k_{\Delta_{12}}, \omega_{\Gamma_{\phi_{12}}}\right)\right), \\
& \mathbb{M H}_{\Lambda_{23}}\left(\phi_{23}\right) \simeq R \Gamma_{\delta_{T^{*} X_{23}}^{a} \Lambda_{23}}\left(T^{*} X_{2233} ; \mu \operatorname{hom}\left(k_{\Delta_{23}}, \omega_{\Gamma_{\phi_{23}}}\right)\right),
\end{aligned}
$$

we obtain a chain of morphisms

$$
\begin{aligned}
& \mathbb{M H}_{\Lambda_{12}}\left(\phi_{12}\right) \otimes \mathbb{M H}_{\Lambda_{23}}\left(\phi_{23}\right) \\
\rightarrow & R \Gamma_{\delta_{T^{*} X_{12}}^{a} \Lambda_{12}{ }_{2}^{a} \delta_{T^{*} X_{23}}^{a} \Lambda_{23}}\left(T^{*} X_{1133} ; \mu h o m\left(k_{\Delta_{12}}, \omega_{\Gamma_{\phi_{12}}}\right) \underset{22}{\stackrel{a}{\circ}} \mu h o m\left(k_{\Delta_{23}}, \omega_{\Gamma_{\phi_{23}}}\right)\right) \\
\rightarrow & \operatorname{R\Gamma }_{\delta_{T^{*} X_{13}}^{a} \Lambda_{13}}\left(T^{*} X_{1133} ; \mu h o m\left(k_{\Delta_{13}}, \omega_{\Gamma_{\phi_{13}}}\right)\right) \\
\simeq & \mathbb{M H}_{\Lambda_{13}}\left(\phi_{13}\right) .
\end{aligned}
$$

Here, the first morphism comes from the assumption (3.3) and the second one is given by Proposition 3.3 ,

\subsection{Microlocal Lefschetz classes of graph trace kernels}

Let $\phi: X \rightarrow X$ be a morphism of manifolds.

Definition 3.5. A $\phi$-graph trace kernel $(K, u, v)$ is the data of $K \in \mathrm{D}^{\mathrm{b}}\left(k_{X \times X}\right)$ together with morphisms

$$
k_{\Delta} \stackrel{u}{\longrightarrow} K \quad \text { and } \quad K \stackrel{v}{\longrightarrow} \omega_{\Gamma_{\phi}} .
$$

In particular, the original trace kernels defined in [KS14 are $\mathrm{id}_{X}$-graph trace kernels. If there is no risk of confusion, we simply write $K$ instead of $(K, u, v)$.

For a $\phi$-graph trace kernel $K$, we set

$$
\begin{aligned}
\mathrm{SS}_{\Delta, \phi}(K): & =\mathrm{SS}(K) \cap T_{\Delta}^{*}(X \times X) \cap T_{\Gamma_{\phi}}^{*}(X \times X) \\
& =\left(\delta_{T^{*} X}^{a}\right)^{-1}\left(\mathrm{SS}(K) \cap T_{\Gamma_{\phi}}^{*}(X \times X)\right) .
\end{aligned}
$$

Definition 3.6. Let $(K, u, v)$ be a $\phi$-graph trace kernel.

(i) The morphism $u$ defines an element $\widetilde{u}$ in $H_{\mathrm{SS}(K) \cap T_{\Delta}^{*}(X \times X)}^{0}\left(T^{*} X ; \mu h o m\left(k_{\Delta}, K\right)\right)$. The microlocal Lefschetz class $\mu \operatorname{Le}(K, \phi) \in H_{\mathrm{SS}_{\Delta, \phi}(K)}^{0}\left(T^{*} X ; \mu h o m\left(k_{\Delta}, \omega_{\Gamma_{\phi}}\right)\right)$ of $K$ is the image of $\widetilde{u}$ under the morphism $\mu h o m\left(k_{\Delta}, K\right) \rightarrow \mu h o m\left(k_{\Delta}, \omega_{\Gamma_{\phi}}\right)$ associated with $v$.

(ii) Let $\Lambda \subset T^{*} X$ be a closed conic subset containing $\operatorname{SS}_{\Delta, \phi}(K)$. We denote by $\mu \operatorname{Le}_{\Lambda}(K, \phi)$ the image of $\widetilde{u}$ in $H_{\Lambda}^{0}\left(T^{*} X ; \mu h o m\left(k_{\Delta}, \omega_{\Gamma_{\phi}}\right)\right)$.

Therefore, we have

$$
\mu \operatorname{Le}_{\Lambda}(K, \phi) \in \mathbb{M H}_{\Lambda}^{0}(\phi)
$$

If there is no risk of confusion, we simply write $\mu \operatorname{Le}(K, \phi)$ instead of $\mu \operatorname{Le}_{\Lambda}(K, \phi)$. 
We denote by $\operatorname{Le}(K, \phi)$ the restriction of $\mu \operatorname{Le}(K, \phi)$ to zero-section $X$ of $T^{*} X$ and call it the Lefschetz class of $K$. Note that

$$
\begin{aligned}
\left.\operatorname{\mu hom}\left(k_{\Delta}, \omega_{\Gamma_{\phi}}\right)\right|_{(X \times X)} & \simeq \operatorname{R} \mathscr{H o m}\left(k_{\Delta}, \omega_{\Gamma_{\phi}}\right) \\
& \simeq\left(\delta_{\phi}\right)_{*} \operatorname{R} \mathscr{H} o m\left(k_{\left(\delta_{\phi}\right)^{-1}(\Delta)}, \omega_{X}\right) \\
& \simeq\left(\delta_{\phi}\right)_{*} \operatorname{R} \Gamma_{M}\left(\omega_{X}\right),
\end{aligned}
$$

where $M:=\{x \in X ; \phi(x)=x\}$ is the fixed point set of $\phi$. Since $\mathrm{R} \Gamma_{M}\left(\omega_{X}\right) \simeq$ $\delta^{-1}\left(\delta_{\phi}\right)_{*} \mathrm{R} \Gamma_{M}\left(\omega_{X}\right)$, we have

$$
\operatorname{Le}(K, \phi) \in H_{M}^{0}\left(X ; \omega_{X}\right)
$$

Graph trace kernels for constructible sheaves. Denote by $\mathrm{D}_{\mathrm{cc}}^{\mathrm{b}}\left(K_{X}\right)$ the full triangulated subcategory of $\mathrm{D}^{\mathrm{b}}\left(k_{X}\right)$ consisting of cohomologically constructible sheaves (see [KS90, Section 3.4]).

Lemma 3.7. Let $F \in \mathrm{D}_{\mathrm{cc}}^{\mathrm{b}}\left(k_{X}\right)$ and $\Phi: \phi^{-1} F \rightarrow F$ be a morphism in $\mathrm{D}_{\mathrm{cc}}^{\mathrm{b}}\left(k_{X}\right)$. There exist natural morphisms in $\mathrm{D}_{\mathrm{cc}}^{\mathrm{b}}\left(k_{X \times X}\right)$

$$
\begin{aligned}
& k_{\Delta} \rightarrow \mathrm{D}_{X} F \otimes F, \\
& \mathrm{D}_{X} F \otimes F \rightarrow \omega_{\Gamma_{\phi}} .
\end{aligned}
$$

In other words, a pair $(F, \Phi)$ of an object $F \in \mathrm{D}_{\mathrm{cc}}^{\mathrm{b}}\left(k_{X}\right)$ and a morphism $\Phi: \phi^{-1} F \rightarrow F$ defines naturally a $\phi$-graph trace kernel.

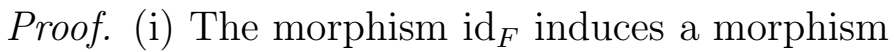

$$
k_{X} \rightarrow \mathrm{R} \mathscr{H} o m(F, F) \simeq \delta^{!}\left(\mathrm{D}_{X} F \otimes F\right) .
$$

Hence, (3.11) is obtained by adjunction.

(ii) Noticing that $\delta_{\phi}^{-1}\left(\mathrm{D}_{X} F \otimes F\right) \simeq \mathrm{D}_{X} F \otimes \phi^{-1} F$, we have a chain of morphisms

$$
\delta_{\phi}^{-1}\left(\mathrm{D}_{X} F \otimes F\right) \stackrel{\Phi}{\rightarrow} \mathrm{D}_{X} F \otimes F \rightarrow \omega_{X} .
$$

Therefore, (3.12) is obtained by adjunction.

We denote by $\operatorname{TK}_{\phi}(F, \Phi)$ the $\phi$-graph trace kernel associated with the pair $(F, \Phi)$ of $F \in \mathrm{D}_{\mathrm{cc}}^{\mathrm{b}}\left(k_{X}\right)$ and $\Phi: \phi^{-1} F \rightarrow F$. The graph trace kernel defines a microlocal Lefschetz class $\mu \operatorname{Le}\left(\operatorname{TK}_{\phi}(F, \Phi), \phi\right)$. We also denote this class by $\mu \operatorname{Le}(F, \Phi, \phi)$. Note that this construction coincides with that of Lefschetz cycles in [MT10].

Graph trace kernels over one point. Let $X=$ pt. In this case, a (graph) trace kernel $(K, u, v)$ is the data of $K \in \mathrm{D}^{\mathrm{b}}(k)$ and morphisms

$$
k \stackrel{u}{\longrightarrow} K \stackrel{v}{\longrightarrow} k .
$$

The (microlocal) Lefschetz class $\operatorname{Le}(K)$ of $K$ is the image of $1 \in k$ under the morphism vu. 
Let us denote by $\mathrm{D}_{f}^{\mathrm{b}}(k)$ the full triangulated subcategory of $\mathrm{D}^{\mathrm{b}}(k)$ consisting of objects with finite-dimensional cohomology. Let $V \in \mathrm{D}_{f}^{\mathrm{b}}(k)$ and $f: V \rightarrow V$ be a $k$-linear map. Set $K:=V^{*} \otimes V$ where $V^{*}:=\operatorname{RHom}(V, k)$. Let $u$ be the dual of the trace morphism $V \otimes V^{*} \rightarrow k$ and $v$ be the composition of $\operatorname{id}_{V^{*}} \otimes f: V^{*} \otimes V \rightarrow V^{*} \otimes V$ and the trace morphism. Then

$$
\operatorname{Le}\left(V^{*} \otimes V\right)=\operatorname{tr}(f):=\sum_{p \in \mathbb{Z}}(-1)^{p} \operatorname{tr}\left(H^{p}(f)\right) .
$$

\section{Main results}

\subsection{Compositions of microlocal Lefschetz classes}

Let $X_{1}, X_{2}, X_{3}$ be manifolds and $\phi_{i}: X_{i} \rightarrow X_{i}(i=1,2,3)$ be morphisms. For $i j=12,23$, let $K_{i j}$ be a $\phi_{i j}$-graph trace kernel.

Lemma 4.1. There are natural morphisms

$$
\begin{gathered}
K_{12} \underset{22}{\circ}\left(k_{\Gamma_{\phi_{2}}} \otimes \omega_{\Gamma_{\phi_{3}}}\right) \rightarrow \omega_{\Gamma_{\phi_{13}}}, \\
k_{\Delta_{13}} \rightarrow K_{12} \underset{22}{*}\left(\omega_{\Delta_{2}}^{\otimes-1} \otimes k_{\Delta_{3}}\right) .
\end{gathered}
$$

Proof. (i) By Lemma 3.2 (i), we have a morphism

$$
\omega_{\Gamma_{\phi_{12} 22} \circ}\left(k_{\Gamma_{\phi_{2}}} \otimes \omega_{\Gamma_{\phi_{3}}}\right) \rightarrow \omega_{\Gamma_{\phi_{13}}} .
$$

Composing it with the morphism $K_{12} \rightarrow \omega_{\Gamma_{\phi_{12}}}$, we get (4.1).

(ii) By Lemma 3.2 (ii), we have a morphism

$$
k_{\Delta_{13}} \rightarrow k_{\Delta_{12}} \underset{22}{*}\left(\omega_{\Delta_{2}}^{\otimes-1} \otimes k_{\Delta_{3}}\right) .
$$

Composing it with the morphism $k_{\Delta_{12}} \rightarrow K_{12}$, we get the morphism (4.2).

Let $\Lambda_{1122} \subset T^{*} X_{1122}$ be a closed conic subset containing $\operatorname{SS}\left(K_{12}\right)$ and $\Lambda_{23}$ be a closed conic subset of $T^{*} X_{23}$. Assume that

$$
\Lambda_{1122} \underset{22}{\stackrel{a}{\times}} \delta_{T^{*} X_{23}}^{a} \Lambda_{23} \rightarrow T^{*} X_{1133} \text { is proper. }
$$

Set

$$
\begin{aligned}
& \Lambda_{12}:=\Lambda_{1122} \cap T_{\Delta_{12}}^{*} X_{1122} \cap T_{\Gamma_{\phi_{12}}}^{*} X_{1122}, \\
& \Lambda_{1133}:=\left(\Lambda_{1122} \cap T_{\Gamma_{\phi_{12}}}^{*} X_{1122}\right) \underset{22}{\stackrel{a}{\circ}} \delta_{T^{*} X_{23}}^{a} \Lambda_{23} \text {, } \\
& \Lambda_{13}:=\Lambda_{1133} \cap T_{\Delta_{13}}^{*} X_{1133} \cap T_{\Gamma_{\phi_{13}}}^{*} X_{1133} \\
& =\Lambda_{12} \stackrel{a}{\stackrel{a}{\circ}} \Lambda_{23} \cap\left(\delta_{T^{*} X_{13}}^{a}\right)^{-1}\left(T_{\Gamma_{\phi_{13}}^{*}}^{*} X_{13}\right) \text {. }
\end{aligned}
$$

We define a map

$$
\Phi_{K_{12}}: \mathbb{M H}_{\Lambda_{23}}\left(\phi_{23}\right) \rightarrow \mathbb{M H}_{\Lambda_{13}}\left(\phi_{13}\right)
$$


by the chain of morphisms

$$
\begin{aligned}
& \mathbb{M H}_{\Lambda_{23}}\left(\phi_{23}\right) \\
& \simeq \mathrm{R} \Gamma_{\delta_{T^{*} X_{23}}^{a} \Lambda_{23}}\left(T^{*} X_{2233} ; \operatorname{\mu hom}\left(k_{\Delta_{23}}, \omega_{\Gamma_{\phi_{23}}}\right)\right) \\
& \simeq R \Gamma_{\delta_{T^{*} X_{23}}^{a} \Lambda_{23}}\left(T^{*} X_{2233} ; \mu h o m\left(\omega_{\Delta_{2}}^{\otimes-1} \otimes k_{\Delta_{3}}, k_{\Gamma_{\phi_{2}}} \otimes \omega_{\Gamma_{\phi_{3}}}\right)\right) \\
& \rightarrow \mathrm{R} \Gamma_{\Lambda_{1133}}\left(T^{*} X_{1133} ; \mu h o m\left(K_{12}, \omega_{\Gamma_{\phi_{12}}}\right) \stackrel{\stackrel{\circ}{\circ}}{\stackrel{a}{2}} \operatorname{\mu hom}\left(\omega_{\Delta_{2}}^{\otimes-1} \otimes k_{\Delta_{3}}, k_{\Gamma_{\phi_{2}}} \otimes \omega_{\Gamma_{\phi_{3}}}\right)\right) \\
& \rightarrow R \Gamma_{\Lambda_{1133}}\left(T^{*} X_{1133} ; \mu h o m\left(K_{12} \underset{22}{*}\left(\omega_{\Delta_{2}}^{\otimes-1} \otimes k_{\Delta_{3}}\right), \omega_{\Gamma_{\phi_{12}}} \underset{22}{\circ}\left(k_{\Gamma_{\phi_{2}}} \otimes \omega_{\Gamma_{\phi_{3}}}\right)\right)\right) \\
& \rightarrow \mathrm{R}_{\delta_{T^{*} X_{13}}^{a} \Lambda_{13}}\left(T^{*} X_{1133} ; \mu h o m\left(k_{\Delta_{13}}, \omega_{\Gamma_{\phi_{13}}}\right)\right) \simeq \mathbb{M H}_{\Lambda_{13}}\left(\phi_{13}\right) \text {. }
\end{aligned}
$$

The first morphism is given by $v: K_{12} \rightarrow \omega_{\Gamma_{\phi_{12}}}$ as follows:

$$
\begin{aligned}
& \mathrm{R} \Gamma_{\delta_{T^{*} X_{23}}^{a} \Lambda_{23}}\left(T^{*} X_{2233} ; \mu h o m(F, G)\right) \\
& \rightarrow \mathrm{R} \Gamma_{\Lambda_{1122} \cap T_{\Gamma_{\phi_{12}}}^{*} X_{1122}}\left(T^{*} X_{1122} ; \mu h o m\left(K, \omega_{\Gamma_{\phi_{12}}}\right)\right) \otimes \mathrm{R}_{\delta_{T^{*} X_{23}}^{a} \Lambda_{23}}\left(T^{*} X_{2233} ; \mu h o m(F, G)\right) \\
& \rightarrow \mathrm{R}_{\Lambda_{1133}}\left(T^{*} X_{1133} ; \mu \operatorname{hom}\left(K_{12}, \omega_{\Gamma_{\phi_{12}}}\right) \stackrel{a}{\stackrel{a}{2}} \mu \operatorname{hom}(F, G)\right) \text {. }
\end{aligned}
$$

Here, we set $F:=\omega_{\Delta_{2}}^{\otimes-1} \otimes k_{\Delta_{3}}, G:=k_{\Gamma_{\phi_{2}}} \otimes \omega_{\Gamma_{\phi_{3}}}$, and $K:=K_{12}$ for simplicity, and use (4.5). The second morphism comes from Proposition 2.5 and the last one is induced by the morphisms in Lemma 3.2 and Lemma 4.1 .

Lemma 4.2. In the situation as above, we have

$$
\Phi_{K_{12}}=\mu \operatorname{Le}\left(K_{12}, \phi_{12}\right)_{2}^{a}: \mathbb{M H}_{\Lambda_{23}}\left(\phi_{23}\right) \rightarrow \mathbb{M H}_{\Lambda_{13}}\left(\phi_{13}\right),
$$

where the right hand side is the map given by Corollary 3.4 .

Proof. Consider the following commutative diagram, where we use the same notation as above: $F:=\omega_{\Delta_{2}}^{\otimes-1} \otimes k_{\Delta_{3}}, G:=k_{\Gamma_{\phi_{2}}} \otimes \omega_{\Gamma_{\phi_{3}}}$ and $K:=K_{12}$.

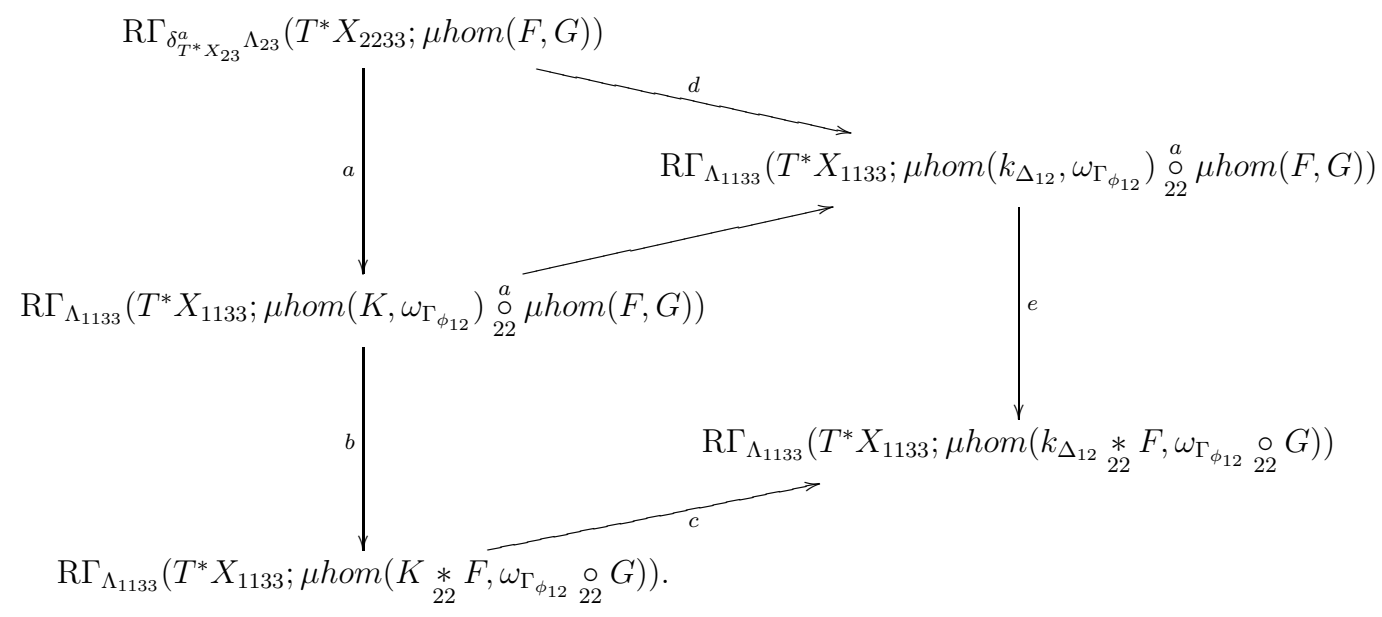

By using the morphisms in Lemma 3.2 , we get a morphism

$$
\begin{aligned}
& w: \mathrm{R}_{\Lambda_{1133}}\left(T^{*} X_{1133} ; \mu h o m\left(k_{\Delta_{12}} \underset{22}{*} F, \omega_{\phi_{12}} \underset{22}{\circ} G\right)\right) \\
& \rightarrow \mathrm{R} \Gamma_{\delta_{T^{*} X_{13}}^{a} \Lambda_{13}}\left(T^{*} X_{1133} ; \mu h o m\left(k_{\Delta_{13}}, \omega_{\phi_{13}}\right)\right) .
\end{aligned}
$$


By construction, the morphism $\Phi_{K_{12}}$ is obtained as the composition of $a, b, c$, and $w$. On the other hand, the morphism $\mu \operatorname{Le}\left(K_{12}, \phi_{12}\right)_{2}^{a}$ is obtained as the composition of $d, e$, and $w$. Hence, the result follows from the commutativity of the above diagram.

For $i j=12,23$, let $\Lambda_{i i j j} \subset T^{*} X_{i i j j}$ be a closed conic subset containing $\operatorname{SS}\left(K_{i j}\right)$. Assume that

$$
\Lambda_{1122} \underset{22}{\stackrel{a}{\times}} \Lambda_{2233} \rightarrow T^{*} X_{1133} \text { is proper. }
$$

Set $\Lambda_{1133}=\Lambda_{1122} \underset{22}{\stackrel{a}{\circ}} \Lambda_{2233}$ and $\Lambda_{13}=\Lambda_{1133} \cap T_{\Delta_{13}}^{*} X_{1133} \cap T_{\Gamma_{\phi_{13}}}^{*} X_{1133}$.

Theorem 4.3. Let $K_{i j}$ be a $\phi_{i j}$-graph trace kernel with $\operatorname{SS}\left(K_{i j}\right) \subset \Lambda_{i i j j}$. Assume that (4.9) holds and set $\widetilde{K}_{23}:=\left(\omega_{2}^{\otimes-1} \otimes k_{233}\right) \otimes K_{23}$. Then the following hold.

(i) The object $K_{12} \underset{22}{\circ} \widetilde{K}_{23}$ is a $\phi_{13}$-graph trace kernel.

(ii) We have $\mu \operatorname{Le}\left(K_{12} \underset{22}{\circ} \widetilde{K}_{23}, \phi_{13}\right)=\mu \operatorname{Le}\left(K_{12}, \phi_{12}\right) \underset{2}{\stackrel{a}{\circ}} \mu \operatorname{Le}\left(K_{23}, \phi_{23}\right)$ as an element of $\mathbb{M H}_{\Lambda_{13}}^{0}\left(\phi_{13}\right)$.

Proof. (i) Tensoring the sequence

$$
k_{\Delta_{23}} \rightarrow K_{23} \rightarrow \omega_{\Gamma_{\phi_{23}}}
$$

with $\omega_{2}^{\otimes-1} \otimes k_{233}$, we get a sequence

$$
\omega_{\Delta_{2}}^{\otimes-1} \otimes k_{\Delta_{3}} \rightarrow \widetilde{K}_{23} \rightarrow k_{\Gamma_{\phi_{2}}} \otimes \omega_{\Gamma_{\phi_{3}}}
$$

Combining this with Lemma 4.1, we have the sequences

$$
k_{\Delta_{13}} \rightarrow K_{12} \underset{22}{*}\left(\omega_{\Delta_{2}}^{\otimes-1} \otimes k_{\Delta_{3}}\right) \rightarrow K_{12} \underset{22}{*} \widetilde{K}_{23}
$$

and

$$
K_{12} \underset{22}{\circ} \widetilde{K}_{23} \rightarrow K_{12} \underset{22}{\circ}\left(k_{\Gamma_{\phi_{2}}} \otimes \omega_{\Gamma_{\phi_{3}}}\right) \rightarrow \omega_{\Gamma_{\phi_{13}}} .
$$

By the assumption (4.9), we have an isomorphism

$$
\alpha: K_{12} \underset{22}{\circ} \widetilde{K}_{23} \stackrel{\sim}{\longrightarrow} K_{12} * \widetilde{K}_{22} .
$$

Using (4.12)-(4.14), we get that $K_{12} \underset{22}{\circ} \widetilde{K}_{23}$ is a $\phi_{13}$-graph trace kernel.

(ii) By Proposition 2.5, under the assumption (4.9), $\operatorname{id}_{K_{12}}$ and $\operatorname{id}_{\widetilde{K}_{23}}$ define a morphism

$$
\beta: K_{12} \underset{22}{*} \widetilde{K}_{23} \rightarrow K_{12} \underset{22}{\circ} \widetilde{K}_{23} .
$$

This morphism is the inverse of the morphism $\alpha$ of (4.14). 
Now let us consider the following commutative diagram:

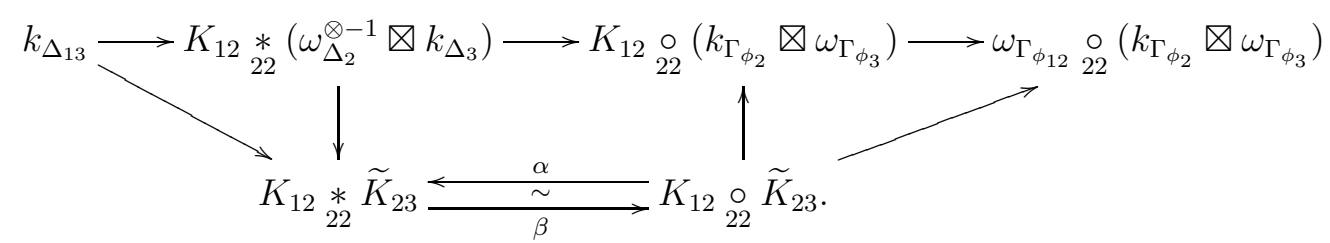

By the graph trace kernel structure of $K_{12} \underset{22}{\circ} \widetilde{K}_{23}$, the composition of the bottom arrows and $\gamma: \omega_{\Gamma_{\phi_{12}}} \underset{22}{\circ}\left(k_{\Gamma_{\phi_{2}}} \otimes \omega_{\Gamma_{\phi_{3}}}\right) \rightarrow \omega_{\Gamma_{\phi_{13}}}$ defines $\mu \operatorname{Le}\left(K_{12} \underset{22}{\circ} \widetilde{K}_{23}, \phi_{13}\right)$. By the construction of the map $\Phi_{K_{12}}$, the composition of the top arrows and $\gamma$ defines $\Phi_{K_{12}}\left(\mu \operatorname{Le}\left(K_{23}, \phi_{23}\right)\right)$. Hence, the result follows from Lemma 4.2,

\subsection{Operations on microlocal Lefschetz classes}

Let $X_{1}$ and $X_{2}$ be manifolds and $\phi_{1}: X_{1} \rightarrow X_{1}$ and $\phi_{2}: X_{2} \rightarrow X_{2}$ be morphisms of manifolds. For $i=1,2$, let $K_{i}$ be a $\phi_{i}$-graph trace kernel and let $\Lambda_{i i}$ be a closed conic subset of $T^{*} X_{i i}$ with $\operatorname{SS}\left(K_{i}\right) \subset \Lambda_{i i}$.

Let $f: X_{1} \rightarrow X_{2}$ be a morphism of manifolds. Assume that $\phi_{2} f=f \phi_{1}$, that is, the diagram

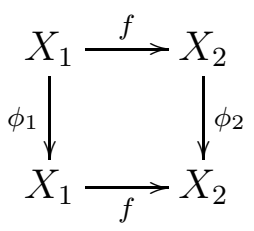

commutes. Since $\Gamma_{f} \subset\left(\phi_{12}\right)^{-1}\left(\Gamma_{f}\right)$, we have a natural morphism

$$
\Phi:\left(\phi_{12}\right)^{-1} k_{\Gamma_{f}} \rightarrow k_{\Gamma_{f}}
$$

Then the pair $\left(k_{\Gamma_{f}}, \Phi\right)$ defines naturally a $\phi_{12}$-graph trace kernel $\operatorname{TK}_{\phi_{12}}\left(k_{\Gamma_{f}}, \Phi\right)$ by Lemma 3.7.

Set $\widetilde{f}:=f \times f: X_{11} \rightarrow X_{22}$. We identify $X_{1212}$ with $X_{1122}$. Then we have

$$
\begin{aligned}
\left(\omega_{1}^{\otimes-1} \otimes k_{122}\right) \otimes \mathrm{TK}_{\phi_{12}}\left(k_{\Gamma_{f}}, \Phi\right) & \simeq\left(\omega_{1}^{\otimes-1} \otimes k_{122}\right) \otimes \omega_{\Gamma_{f}} \otimes k_{\Gamma_{f}} \\
& \simeq k_{\Gamma_{f}} \otimes k_{\Gamma_{f}} \\
& \simeq k_{\Gamma_{\tilde{f}}}
\end{aligned}
$$

We also note that

$$
\mathrm{R} \widetilde{f_{!}} K_{1} \simeq K_{1} \circ k_{\Gamma_{\tilde{f}}}, \quad \widetilde{f}^{-1} K_{2} \simeq k_{\Gamma_{\widetilde{f}} \circ 2} K_{2}
$$

External product. Let $X_{2}=$ pt. We then write $X_{2}$ instead of $X_{3}$. For $i=1,2$, let $\Lambda_{i}$ be a closed conic subset of $T^{*} X_{i}$. In this case, we have the composition morphism

$$
\mathbb{M H}_{\Lambda_{1}}\left(\phi_{1}\right) \otimes \mathbb{M H}_{\Lambda_{2}}\left(\phi_{2}\right) \stackrel{\circ}{\longrightarrow} \mathbb{M H}_{\Lambda_{1} \times \Lambda_{2}}\left(\phi_{12}\right) .
$$


Taking the 0-th cohomology, we have a morphism

$$
\mathbb{M H H}_{\Lambda_{1}}^{0}\left(\phi_{1}\right) \otimes \mathbb{M H}_{\Lambda_{2}}^{0}\left(\phi_{2}\right) \stackrel{\circ}{\longrightarrow} \mathbb{M H}_{\Lambda_{1} \times \Lambda_{2}}^{0}\left(\phi_{12}\right) .
$$

In this case, we shall denote by $\lambda_{1} \times \lambda_{2}$ instead of $\lambda_{1} \circ \lambda_{2}$.

Set

$$
\Lambda_{i}:=\Lambda_{i i} \cap T_{\Delta_{i}}^{*} X_{i i} \quad(i=1,2) .
$$

Then by Theorem 4.3, we obtain the following.

Proposition 4.4. The object $K_{1} \otimes K_{2}$ is a $\phi_{12}$-graph trace kernel and

$$
\mu \operatorname{Le}\left(K_{1} \otimes K_{2}, \phi_{12}\right)=\mu \operatorname{Le}\left(K_{1}, \phi_{1}\right) \times \mu \operatorname{Le}\left(K_{2}, \phi_{2}\right) .
$$

Direct image. Let $X_{1}=$ pt. We then write $X_{1}, X_{2}$ instead of $X_{2}, X_{3}$. Let $\Lambda_{1} \subset$ $T^{*} X_{1}$ be a closed conic subset. Assume that

$$
f \text { is proper on } \Lambda_{1} \cap T_{X_{1}}^{*} X_{1} \text {. }
$$

We set

$$
\begin{aligned}
f_{\mu, \phi_{1} \rightarrow \phi_{2}}\left(\Lambda_{1}\right): & =\Lambda_{1} \stackrel{a}{\circ} T_{\Gamma_{f}}^{*} X_{12} \cap\left(\delta_{T^{*} X_{2}}^{a}\right)^{-1}\left(T_{\Gamma_{\phi_{2}}}^{*} X_{22}\right) \\
& =f_{d} f_{\pi}^{-1}\left(\Lambda_{1}\right) \cap\left(\delta_{T^{*} X_{2}}^{a}\right)^{-1}\left(T_{\Gamma_{\phi_{2}}}^{*} X_{22}\right) \subset T^{*} X_{2},
\end{aligned}
$$

and

$$
f_{\mu, \phi_{1} \rightarrow \phi_{2}}:=\stackrel{a}{\circ} \mu \operatorname{Le}\left(k_{\Gamma_{f}}, \Phi, \phi_{12}\right): \mathbb{M H}_{\Lambda_{1}}^{0}\left(\phi_{1}\right) \rightarrow \mathbb{M H}_{f_{\mu, \phi_{1} \rightarrow \phi_{2}}\left(\Lambda_{1}\right)}^{0}\left(\phi_{2}\right) .
$$

Proposition 4.5. Assume that $\tilde{f}$ is proper on $\Lambda_{11} \cap T_{X_{11}}^{*} X_{11}$ and set $\Lambda_{1}:=\Lambda_{11} \cap$ $T_{\Delta_{1}}^{*} X_{11}$. Then the object $\mathrm{R} \widetilde{f}_{!} K_{1}$ is a $\phi_{2}$-graph trace kernel and

$$
\mu \operatorname{Le}\left(\mathrm{R} \tilde{f}_{!} K_{1}, \phi_{2}\right)=f_{\mu, \phi_{1} \rightarrow \phi_{2}}\left(\mu \operatorname{Le}\left(K_{1}, \phi_{1}\right)\right) .
$$

Proof. The assumption implies that $\Lambda_{11} \underset{11}{\stackrel{a}{\times}} T_{\Gamma_{\tilde{f}}}^{*} X_{1122} \rightarrow T^{*} X_{22}$ is proper. By Theorem 4.3. $K_{1} \circ\left(\omega_{1}^{\otimes-1} \otimes k_{122}\right) \otimes \mathrm{TK}_{\phi_{12}}\left(k_{\Gamma_{f}}, \Phi\right) \simeq K_{1} \circ k_{\Gamma_{\tilde{f}}} \simeq \mathrm{R} \widetilde{f}_{!} K_{1}$ is a $\phi_{2}$-graph trace kernel and we have

$$
\mu \operatorname{Le}\left(\mathrm{R} \widetilde{f}_{!} K_{1}, \phi_{2}\right)=\mu \operatorname{Le}\left(K_{1}, \phi_{1}\right) \stackrel{a}{\circ} \mu \operatorname{Le}\left(k_{\Gamma_{f}}, \Phi, \phi_{12}\right) .
$$

Inverse image. Let $X_{3}=$ pt. Let $\Lambda_{2} \subset T^{*} X_{2}$ be a closed conic subset. Assume that

$$
f \text { is non-characteristic for } \Lambda_{2} \text {. }
$$

We set

$$
\begin{aligned}
f^{\mu, \phi_{1} \rightarrow \phi_{2}}\left(\Lambda_{2}\right): & =T_{\Gamma_{f}}^{*} X_{12} \stackrel{a}{\stackrel{a}{\circ}} \Lambda_{2} \cap\left(\delta_{T^{*} X_{1}}^{a}\right)^{-1}\left(T_{\Gamma_{\phi_{1}}}^{*} X_{11}\right) \\
& =f_{d} f_{\pi}^{-1}\left(\Lambda_{2}\right) \cap\left(\delta_{T^{*} X_{1}}^{a}\right)^{-1}\left(T_{\Gamma_{\phi_{1}}}^{*} X_{11}\right) \subset T^{*} X_{1},
\end{aligned}
$$

and

$$
f^{\mu, \phi_{1} \rightarrow \phi_{2}}:=\mu \operatorname{Le}\left(k_{\Gamma_{f}}, \Phi, \phi_{12}\right)_{2}^{a}: \mathbb{M H}_{\Lambda_{2}}^{0}\left(\phi_{2}\right) \rightarrow \mathbb{M H}_{f^{\mu, \phi_{1} \rightarrow \phi_{2}\left(\Lambda_{2}\right)}}^{0}\left(\phi_{1}\right)
$$


Proposition 4.6. Assume that $\tilde{f}$ is non-characteristic for $\Lambda_{22}$ and set $\Lambda_{2}:=\Lambda_{22} \cap$ $T_{\Delta_{2}}^{*} X_{22}$. Then the object $\left(\omega_{X_{1} / X_{2}} \otimes k_{1}\right) \otimes \widetilde{f}^{-1} K_{2}$ is a $\phi_{1}$-graph trace kernel and

$$
\mu \operatorname{Le}\left(\left(\omega_{X_{1} / X_{2}} \otimes k_{1}\right) \otimes \tilde{f}^{-1} K_{2}, \phi_{1}\right)=f^{\mu, \phi_{1} \rightarrow \phi_{2}}\left(\mu \operatorname{Le}\left(K_{2}, \phi_{2}\right)\right) .
$$

Proof. The assumption implies that $T_{\Gamma_{\tilde{f}}}^{*} X_{1122} \underset{22}{\stackrel{a}{\times}} \Lambda_{22} \rightarrow T^{*} X_{11}$ is proper. By Theorem 4.3. $\mathrm{TK}_{\phi_{12}}\left(k_{\Gamma_{f}}, \Phi\right) \underset{22}{\circ}\left(\omega_{2}^{\otimes-1} \otimes k_{2}\right) \otimes K_{2}$ is a $\phi_{1}$-graph trace kernel. Here, we have isomorphisms

$$
\begin{aligned}
\operatorname{TK}_{\phi_{12}}\left(k_{\Gamma_{f}}, \Phi\right) \underset{22}{\circ}\left(\omega_{2}^{\otimes-1} \otimes k_{2}\right) \otimes K_{2} & \simeq\left(\omega_{1} \otimes k_{1}\right) \otimes\left(k_{\Gamma_{\widetilde{f}} \circ 2}\left(\omega_{2}^{\otimes-1} \otimes k_{2}\right) \otimes K_{2}\right) \\
& \simeq\left(\omega_{1} \otimes k_{1}\right) \otimes \widetilde{f}^{-1}\left(\left(\omega_{2}^{\otimes-1} \otimes k_{2}\right) \otimes K_{2}\right) \\
& \simeq\left(\omega_{1} \otimes k_{1}\right) \otimes\left(f^{-1} \omega_{2}^{\otimes-1} \otimes f^{-1} k_{2}\right) \otimes \widetilde{f}^{-1} K_{2} \\
& \simeq\left(\omega_{X_{1} / X_{2}} \otimes k_{1}\right) \otimes \widetilde{f}^{-1} K_{2} .
\end{aligned}
$$

Applying Theorem 4.3 again, we have

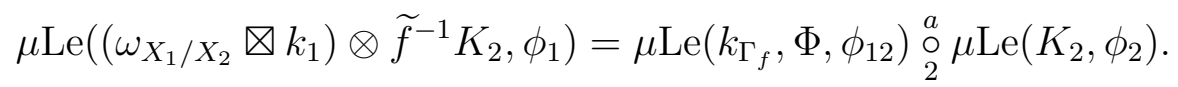

Tensor product. Let $X_{1}=X_{2}=X$ and $\phi_{1}=\phi_{2}=\phi$. For $i=1,2$, let $K_{i}$ be a $\phi$-graph trace kernel and $\Lambda_{i i} \subset T^{*}(X \times X)$ be a closed conic subset satisfying $\mathrm{SS}\left(K_{i}\right) \subset \Lambda_{i i}$. Assume that

$$
\Lambda_{11} \cap \Lambda_{22}^{a} \subset T_{X \times X}^{*}(X \times X),
$$

and set

$$
\Lambda_{i}:=\Lambda_{i i} \cap T_{\Delta}^{*}(X \times X) \quad(i=1,2) .
$$

Recall that for a morphism $f: X \rightarrow Y$, we set $\tilde{f}:=f \times f: X \times X \rightarrow Y \times Y$. Since $\widetilde{\phi} \delta=\delta \phi$, we have a morphism

$$
\delta^{\mu, \phi \rightarrow \widetilde{\phi}}: \mathbb{M H}_{\Lambda_{1} \times \Lambda_{2}}^{0}(\widetilde{\phi}) \rightarrow \mathbb{M H}_{\Lambda_{1}+\Lambda_{2}}^{0}(\phi) .
$$

Composing it with the morphism of external product

$$
\times: \mathbb{M H}_{\Lambda_{1}}^{0}(\phi) \otimes \mathbb{M H}_{\Lambda_{2}}^{0}(\phi) \rightarrow \mathbb{M H}_{\Lambda_{1} \times \Lambda_{2}}^{0}(\widetilde{\phi})
$$

we get a convolution morphism

$$
\star: \mathbb{M H}_{\Lambda_{1}}^{0}(\phi) \otimes \mathbb{M H}_{\Lambda_{2}}^{0}(\phi) \rightarrow \mathbb{M H}_{\Lambda_{1}+\Lambda_{2}}^{0}(\phi) .
$$

Proposition 4.7. Assume that (4.30) holds. Then the object $\left(\omega_{X}^{\otimes-1} \otimes k_{X}\right) \otimes K_{1} \otimes K_{2}$ is a $\phi$-graph trace kernel and

$$
\mu \operatorname{Le}\left(\left(\omega_{X}^{\otimes-1} \otimes k_{X}\right) \otimes K_{1} \otimes K_{2}, \phi\right)=\mu \operatorname{Le}\left(K_{1}, \phi\right) \star \mu \operatorname{Le}\left(K_{2}, \phi\right) .
$$

Proof. Since we regard $\widetilde{\delta}: X \times X \rightarrow X \times X \times X \times X$ as the map $\left(x_{1}, x_{2}\right) \mapsto$ $\left(x_{1}, x_{2}, x_{1}, x_{2}\right)$, we have $\widetilde{\delta}^{-1}\left(K_{1} \otimes K_{2}\right) \simeq K_{1} \otimes K_{2}$. The assumption implies $\delta$ is non-characteristic for $\Lambda_{1} \times \Lambda_{2}$. Thus, the result follows from Proposition 4.4 and 4.6, since $\omega_{X / X \times X} \simeq \omega_{X}^{\otimes-1}$. 


\subsection{Application to Lefschetz fixed point formula for con- structible sheaves}

Let $X$ be a real analytic manifold and $\phi_{X}: X \rightarrow X$ be a morphism of manifolds. We denote by $\mathrm{D}_{\mathbb{R}-\mathrm{c}}^{\mathrm{b}}\left(k_{X}\right)$ the full triangulated subcategory of $\mathrm{D}^{\mathrm{b}}\left(k_{X}\right)$ consisting of $\mathbb{R}$-constructible complexes. Since $\mathbb{R}$-constructible complexes are cohomologically constructible, a pair $(F, \Phi)$ of an object $F \in \mathrm{D}_{\mathbb{R}-\mathrm{c}}^{\mathrm{b}}\left(k_{X}\right)$ and a morphism $\Phi: \phi_{X}^{-1} F \rightarrow F$ gives naturally a $\phi_{X}$-graph trace kernel $\operatorname{TK}_{\phi_{X}}(F, \Phi)$.

Let $Y$ be another real analytic manifold and $\phi_{Y}: Y \rightarrow Y$ be a morphism. Let $f: X \rightarrow Y$ be a morphism of manifolds which satisfies $\phi_{Y} f=f \phi_{X}$. Then we have a natural morphism

$$
\begin{aligned}
\phi_{Y}^{-1} \mathrm{R} f_{*} F & \rightarrow \phi_{Y}^{-1} \mathrm{R} f_{*} \mathrm{R} \phi_{X *} \phi_{X}^{-1} F \\
& \stackrel{\Phi}{\rightarrow} \phi_{Y}^{-1} \mathrm{R} \phi_{Y *} \mathrm{R} f_{*} F \\
& \rightarrow \mathrm{R} f_{*} F .
\end{aligned}
$$

We denote this morphism by $\mathrm{R} f_{*} \Phi$.

Proposition 4.8. Assume that $f$ is proper on $\operatorname{Supp}(F)$. Then

$$
\mu \operatorname{Le}\left(\mathrm{R} f_{*} F, \operatorname{R} f_{*} \Phi, \phi_{Y}\right)=f_{\mu, \phi_{X} \rightarrow \phi_{Y}}\left(\mu \operatorname{Le}\left(F, \Phi, \phi_{X}\right)\right) .
$$

Proof. By assumption, $\mathrm{R} f_{*} F \in \mathrm{D}_{\mathbb{R}-\mathrm{c}}^{\mathrm{b}}\left(k_{Y}\right)$. Hence, the pair $\left(\mathrm{R} f_{*} F, \mathrm{R} f_{*} \Phi\right)$ gives a $\phi_{Y}$-trace kernel $\mathrm{TK}_{\phi_{Y}}\left(\mathrm{R} f_{*} F, \mathrm{R}_{*} \Phi\right)$. Then we have an isomorphism

$$
\mathrm{TK}_{\phi_{Y}}\left(\mathrm{R} f_{*} F, \mathrm{R} f_{*} \Phi\right) \simeq \mathrm{R} \tilde{f}_{!} \operatorname{TK}_{\phi_{X}}(F, \Phi) .
$$

Hence, by Proposition 4.5,

$$
\begin{aligned}
\mu \operatorname{Le}\left(\mathrm{R} f_{*} F, \operatorname{R} f_{*} \Phi, \phi_{Y}\right) & =\mu \operatorname{Le}\left(\mathrm{R} \tilde{f}_{!} \operatorname{TK}_{\phi_{X}}(F, \Phi), \phi_{Y}\right) \\
& =f_{\mu, \phi_{X} \rightarrow \phi_{Y}}\left(\mu \operatorname{Le}\left(F, \Phi, \phi_{X}\right)\right) .
\end{aligned}
$$

Note that the above formula is similar to that of [MT10].

Applying Proposition 4.8 for $Y=\mathrm{pt}$ and the natural morphism $f=\mathbf{a}: X \rightarrow \mathrm{pt}$, we obtain

Corollary 4.9. Assume that $\operatorname{Supp}(F)$ is compact. Then

$$
\operatorname{tr}(F, \Phi)=\mathbf{a}_{\mu}\left(\mu \operatorname{Le}\left(F, \Phi, \phi_{X}\right)\right)
$$

where the left hand side is defined by

$$
\operatorname{tr}(F, \Phi):=\sum_{p \in \mathbb{Z}}(-1)^{p} \operatorname{tr}\left(H^{p}(X ; F) \rightarrow H^{p}\left(X ; \phi_{X}^{-1} F\right) \stackrel{\Phi}{\rightarrow} H^{p}(X ; F)\right) .
$$

\section{Acknowledgments}

The author is grateful to Professor Pierre Schapira for helpful advice. This work was supported by JSPS KAKENHI Grant Number 15J07993 and the Program for Leading Graduate Schools, MEXT, Japan. 


\section{References}

[Gu96] S. Guillermou, Lefschetz class of elliptic pairs, Duke Math. J. 85 (1996), no. 2, 273-314.

[IMT15] Y. Ike, Y. Matsui and K. Takeuchi, Hyperbolic localization and Lefschetz fixed point formulas for higher-dimensional fixed point sets, preprint arXiv:math/1504.04185.

[KS90] M. Kashiwara and P. Schapira, Sheaves on Manifolds, Grundlehren der Math. Wiss. 292, Springer-Verlag, Berlin, 1990.

[KS14] M. Kashiwara and P. Schapira, Microlocal Euler classes and Hochschild homology, J. Inst. Math. Jussieu, 13 (2014), no. 3, 487-516.

[MT10] Y. Matsui and K. Takeuchi, Microlocal study of Lefschetz fixed-point formulas for higher-dimensional fixed point sets, Int. Math. Res. Not. IMRN, (2010), no. 5, 882-913.

[RTT13] A. C. Ramadoss, X. Tang, and H.-H. Tseng, Hochschild Lefschetz class for D-modules, Math. Z. 275 (2013), no. 1-2, 367-388.

[Sc14] P. Schapira, Private communications.

[ScSn94] P. Schapira and J-P. Schneiders, Index Theorem for Elliptic Pairs, Astérisque Soc. Math. France. 224, 1994.

Graduate School of Mathematical Sciences, the University of Tokyo, Komaba, Tokyo, 153-8914, Japan

E-mail address: ike@ms.u-tokyo.ac.jp 